\title{
ESTUDIO DE UN RIO DE MONTAÑA DE UNA ZONA GRANITICA DEL NORTE DE PORTUGAL
}

\author{
M. V. Cortés, Rui (1); K. Tjarda de, (2); M. A. Simoes Graça, (3) \\ (1) Instituto Universitario de Tras-os-Montes e Alto Douro; (2) Instituto Politécnico Bragança Portugal; (3) Dpto- \\ .de Zoología Universidade de Coimbra Portugal.
}

Palabras clave: Biotipology, macroinvertebrates, physico-chemical analises, fishes.

\begin{abstract}
STUDY OF A MOUNTAIN STREAM IN A GRANITIC REGION IN THE NORTH OF PORTUGAL
\end{abstract}

Macrofauna, fishes and physico-chemical characteristics have been studied in the Olo nver (Douro Basin), placed in the Natural Park of Alvão, north Portugal.

During 1.5 year, every 3 months, samples of macrofauna and water for chemical analyses have been taken in 3 stations along the river. In order to assess fish species and biomass, electrofishing has been made in 2 stations.

This stream can be charactenzed as a non-polluted headstream with low concentrations of organic matter and dissolved salts, and a high stability of the chemical parameters all along the year. The chemical data shows no significant difference between the stations.

The fish population has a low biomass. Dominant species are Salmo trutta fario L. and Chondostroma polylepis polylepis S. The macrofauna shows a high diversity and no significant difference betwcen the stations. 177 identification units have been distinguished, and 89 of them are species. Dominant species are Baetis rhodani and Calliarcys humilis in the Ephemeroptera group, Plectrocnemia geniculata and Hydropsyche siltalai in the Tnchoptera one. In Plecoptera Leuctra gr. fusca is dominant.

Stnking differences with data on macrofauna in Spanish parts of Douro Basin are the absence of Baetis gr. alpina in the upstream station, while Tanago (1 984) mentions this group for epirhitron, as well as the replacement of Gammarus sp. by Pro-asellus gr. coxalis. unlike the Jalon and Tanago (1982) data.

\section{INTRODUCCION}

Han sido bastante reducidos los trabajos realizados en Portugal en lo referente a estudios biotipológicos o simplemente en lo que concierne a la uiilización de macroinvertebrados como indicadores biológicos; por otra parte los estudios realizados hasta el momento han abarcado áreas relativamente limitadas: Cortes (1981), Snusa et al. (1984), Fontoura (1984).

El río Olo presenta un interés particular por incidir en un curso de agua que constituye el eje central del recién creado «Parque Natural do Alváo», en el cual, aunque no existan actualmente fuentes de contaminación dignas de registro, está sujeto a una creciente influencia de actividades turísticas, por lo que interesa caracterizar: química y biológicamente este ecosistema con el fin de detectar alteraciones que se vayan a verificar.

Limnética 2: 197-204 (1986)

O Asociación Española de Limnología, Madrid. Spain
Por otra parte, se procuró igualmente comparar los datos biológicos de este río con otros obtenidos por Tanago \& Jalon (1982) en ríos de montaña de la parte española de la cuenca del Duero.

\section{MATERIAL Y METODOS}

Se realizó trimestralmente la recogida de agua y de fauna béntica desde junio de 1983 a septiembre de 1984 en tres estaciones de montaña (Fig. 1). La estación 1 localizada a $6 \mathrm{Km}$. del nacimiento se encuentra a una altitud de $990 \mathrm{~m}$., la estación 2 dista de ella $10 \mathrm{Km}$. y su altitud es de $770 \mathrm{~m}$.; la estación 3 se localiza igualmente a $10 \mathrm{Km}$. de la anterior y está a una altitud de $230 \mathrm{~m}$. El sustrato es pedregoso (piedras de 10 a $40 \mathrm{~cm}$. de diámetro) y muy semejante en todas las estaciones al igual que la profundidad y la 


\begin{tabular}{|c|c|c|c|c|c|c|c|c|c|c|c|}
\hline Est. & $\mathrm{pH}$ & $\begin{array}{r}\text { Alcal. } \\
(\mathrm{meq} / 1)\end{array}$ & $\begin{array}{r}\text { Dureza } \\
\text { (meq./1) }\end{array}$ & $\begin{array}{l}\text { Conduc. } \\
\text { (uohms } \mathrm{cm} \text { ) }\end{array}$ & $\begin{array}{l}0_{2} \text { dis. } \\
\text { (mg./1) }\end{array}$ & $\begin{array}{l}\mathrm{DBO}_{5} \\
\left.\mathrm{~g} .0_{2} / 1\right)\end{array}$ & $\begin{array}{l}\text { D.Q.O. } \\
\text { (mg./1) }\end{array}$ & $\begin{array}{r}\text { Cloruros } \\
\text { (mg./1) }\end{array}$ & $\begin{array}{r}\text { moníaco } \\
(\mathrm{mg} . / 1)\end{array}$ & $\begin{array}{r}\text { Nitratos } \\
\text { (mg./1) }\end{array}$ & $\begin{array}{r}\text { Ortofosf. } \\
(\mathrm{mg} . / 1)\end{array}$ \\
\hline 1 & 6.2 & 0.10 & 0.11 & 18.7 & 9.63 & 1.08 & 6.52 & 2.70 & $<0.1$ & 0.03 & 0.02 \\
\hline & \pm 0.21 & \pm 0.03 & \pm 0.05 & \pm 1.1 & \pm 0.62 & \pm 0.72 & \pm 6.17 & \pm 1.22 & & \pm 0.03 & \pm 0.04 \\
\hline 2 & 6.1 & 0.09 & 0.133 & 27.2 & 9.97 & 1.47 & 9.86 & 2.83 & $<0.1$ & 0.03 & $<0.01$ \\
\hline & \pm 0.26 & \pm 0.04 & \pm 0.03 & \pm 6.5 & \pm 0.89 & \pm 0.42 & \pm 9.95 & \pm 0.75 & & \pm 0.02 & \\
\hline 3 & $\begin{array}{ll} & 6.2 \\
+ & 0.46\end{array}$ & $\begin{array}{r}0.14 \\
+\quad 0.04\end{array}$ & $\begin{array}{r}0.20 \\
\pm \quad 0.09\end{array}$ & $\begin{array}{r}29.4 \\
+\quad 4.0\end{array}$ & $\begin{array}{r}9.90 \\
+\quad 0.96\end{array}$ & $\begin{array}{r}1.38 \\
+\quad 0.42\end{array}$ & $\begin{array}{r}8.32 \\
+\quad 10.85\end{array}$ & $\begin{array}{r}3.48 \\
\pm \quad 0.71\end{array}$ & $<0.1$ & $\begin{array}{r}0.11 \\
\pm \quad 0.07\end{array}$ & $\begin{array}{r}0.01 \\
+0.01\end{array}$ \\
\hline
\end{tabular}

Tabla 1.-Características físico-químicas medias de las aguas del río Olo en las tres estaciones de muestreo. Mean physico-chemical characteristics of Olo river water of the three sampling stations.

\section{EST. $1 / 2 \quad$ EST. $2 / 3 \quad$ EST. $1 / 3$}

$\begin{array}{cccc}\mathrm{n} & 88 & 88 & 88 \\ \mathrm{r}_{\mathrm{s}} & 0.66 & 0.86 & 0.61 \\ \mathrm{t} & 8.10 & 15.29 & 7.08\end{array}$

$\mathrm{n}=$ Número de especies comparadas Number ofcompared species. $\mathrm{t}=$ Valor de $\mathrm{t}$. $\mathrm{t}$ value.

Tabla 2.-Determinación de la similitud entre cada par de estaciones de niuestreo Determination of similarity between each pair of sarnpling stations.

\begin{tabular}{|c|c|c|c|c|c|c|}
\hline & $6 / 83$ & $9 / 83$ & $12 / 83$ & $3 / 84$ & $6 / 84$ & $9 / 84$ \\
\hline Est. 1 & 3.64 & 4.35 & 3.40 & 4.02 & 4.02 & 4.14 \\
\hline Est. 2 & 4.17 & 3.91 & 3.91 & 3.57 & 4.14 & 3.34 \\
\hline Est. 3 & 3.64 & 3.96 & 4.10 & 2.77 & 4.42 & 3.52 \\
\hline
\end{tabular}

Tabla 3.-Indices de diversidad de Shannon-Weaver para las tres estaciones de muestreo para cada fecha.

Diversity indices of Shannon-Weaverfor the three sampling stations each season.

agitación del agua, siendo ésta uniformemente turbulenta. La anchura del río varía de $3,5 \mathrm{~m}$. en la estación 1 a 13 m. en la estación 3.

Los análisis fisico-químicos consisten en la determinación del $\mathrm{pH}$, y conductividad (en el lugar) y dureza, alcalinidad, cloruros, oxígeno disuelto, D.B.O, y D.Q.O. por procesos titulimétricos y amoníaco, nitratos y ortofosfatos por espectrofotometría.

Para las capturas de macroinvertebrados se utilizó una red manual de $670 \mu$, habiendose adoptado un proceso semi-cuantitativo definiendo un tiempo de captura idéntico en todos los casos y procurando muestrear todos los micro-habitats; la separación del material biológico se realizó en el laboratorio y sólo después se procedió a su conservación en alcohol al $70 \%$.

Las capturas de las poblaciones de peces apenas tuvieron lugar en abril de 1985 y únicamente en las es- taciones 1 y 3 . Se recurrió a la pesca eléctrica con corriente continua de $1-2 \mathrm{~A}$ y 440 volts. utilizando un generador portátil de $500 \mathrm{~W}$. habiéndose delimitado previamente con redes sectores de cerca de $50 \mathrm{~m}$.

Para determinar la similitud entre las estaciones a partir de las especies bénticas se calculó el coeficiente de correlación de Spearman $\left(\mathrm{r}_{\mathrm{s}}\right)$. Para conocer la significación de las correlaciones se determinó tal como fue definido por Steel y Torrie (1981) y los valores obtenidos fueron así comparados con los de una lista de distribución de t.

Por su parte la diversidad de todas las muestras fue calculada por el índice de Shannon-Weaver-H'. (1974).

Finalmente la biomasa piscicola por unidad de área se basó en un método indirecto sin marcaje de Lury, referido por Amanieu \& Lassere (1971). 

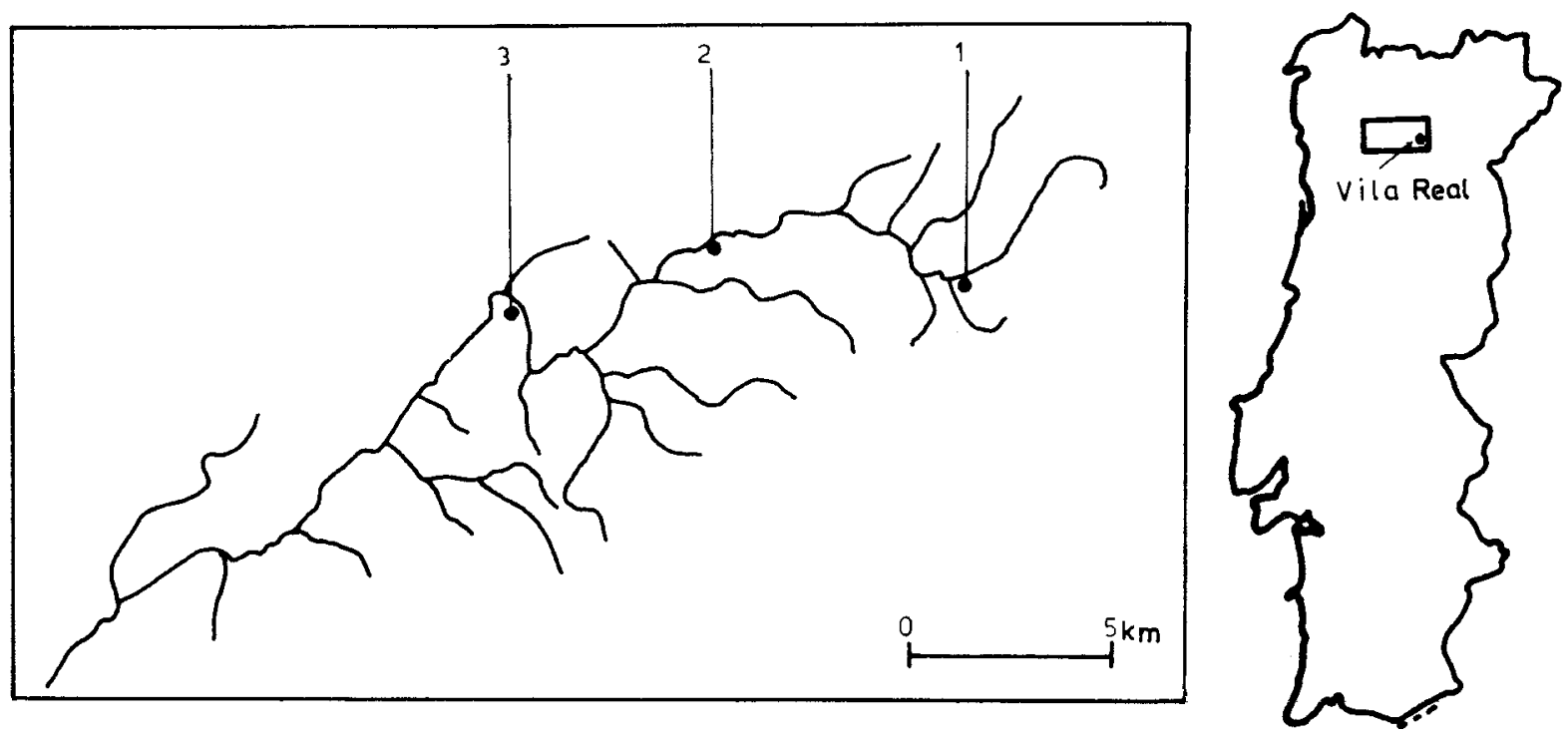

Figura 1.-Localización del no Olo y de las tres estaciones de muestreo. Localization of Olo river and the three sampling stations.

\section{RESULTADOS Y DISCUSION}

Caracterización fisico-quimica.

Los valores medios para cada estación y respectivos intervalos de confianza $(\mathrm{p}>0,01)$ se encuentran expresados en la tabla I. De su análisis pudimos comprobar además una gran semejanza entre las estaciones, así como una acentuada estabilidad química a lo largo del año, el carácter ácido de las aguas del río Olo y su bajo contenido en sales disueltas (como consecuencia de rocas graníticas difícilmente erosionables y de una agricultura que utiliza reducidas cantidades de fertilizantes), y también bajos contenidos en materia orgánica y altos en oxígeno característicos de aguas limpias.

\section{Biotipologia}

En el dpéndice están referidas las especies presentes para cada estación a lo largo del año y medio, expresadas en porcentaje. Según la clasificación de Illies y Botosaneanu (1963), la zona en estudio ocupa fundamentalmente metarhitrón, teniendo en cuenta el declive, la velocidad de la corriente y el tipo de sustrato. He aquí un breve análisis de los principales grupos presentes:

Tricladida: si las especies dominates Polycelis felina y Dugesia gonocephala siguen los esquemas de zonación clásico presentado por Legier (1972) y Baguña et al (1982) con la dominancia de la primera en zonas de mayor altitud. la presencia de Polvcelis gr nigra (englobando $P$. tenuis) en la estación 1 no refleja la ecología normal de esta especie característica de tramos tranquilos (Pattee y Gourbault, 1981).

Trichoptera: en lo que se refiere a la familia Rhyacophilidae. es nítida la restricción de la Rhyacophila adjuncta a las zonas más altas. lo que está además de acuerdo con Jalón (1981) y Jalón y Tanago (1982) observándose idéntica distribución para la $R$. pulchra. En la familia Brachycentridae se nota la nítida preferencia y constancia de Microsema minimum por la mayor altitud en cuanto que M. longulum le sucede: esta distribución corresponde a la mencionada en Decamps (1970). En relación a las familias Limnephilidae y Goeridae la diversidad es superior en la estación 1, ya que las especies como Potamophylax latipennis y Silo graellsi son mas abundantes en las partes altas del río y de baja mineralización. También la especie Odontocerum albicorne (Odontoceridae) está restringida a la estación más elevada. En la familia Hydropsychidae el tipo de distribución de Hydropsyche pellucidula y principalmente de $\mathrm{H}$. siltalai es distinto del mencionado por Boom (1974) y también de Tanago y Jalón (1982), ya que estas especies aumentan de frecuencia hacia la parte inferior del río; de cualquier modo Vernaux y Faersel (1976), así como Lapchin y Ncveu (1979). señalaron su amplio espectro ecológico.

Ephemeroptera: los valores cuantitativos de Ephemerella ignita disminuyen hacia la desembocadura, lo que contradice un estudio efectuado por Guidicelli \& Legier (1980) en Argens (Francia) en que esta cspe- 
cie era considerada representativa de la comunidad de transición rhitron-potamon.

Callyarcis humilis obedece al mismo tipo de variación, ya observado por Tanago (1984a) en la cuenca del Duero; algunas variaciones longitudinales son visibles dentro del gen. Baetis aunque sea siempre $R$. rhodani la especie dominante y la única presente en la estación 1, mientras que B. fuscatus, B. buccratus, $B$. alpinus y $R$. melanonyx aparecen en las estaciones siguientes; conviene destacar la ausencia del grupo alpinus (englobando B. melanonys) en aquella estación. ya que Tanago (1984b) menciona la captura de este grupo fundamentalmente en la zona alta del epirhitron.

Serratella albai, Paraleprophlebia cincta y Dipterophlebiodes sp. son más abundantes en el hyporhythron.

Plecoptera: es de resaltar la preferencia de Euletuctra geniculata por la estación 3, aunque Puig (1984) hubiera capturado esta especie solamente en zonas calcáreas de ríos de Cataluña. o sea, en un medio muy distinto del río Olo.

Al contrario sucede con Amphinemura sp. que presenta mayores cantidades y diversidad en las estaciones 1 y 2 .

Coleoptera: disminución en la diversidad y n." de individuos hacia la desembocadui-a. en este último caso debido a Oulimniuss sp.

Diptera: notable constancia de los grupos dominantes y una relativa poca importancia de los quironómidos en relación a la totalidad del macrobentos, aunque pensemos que la importancia de esta familia haya sido en parte ocultada por la utilización de una red de gran abertura, dando lugar como refiere Resh (1979) a un error frecuente de muestreo que consiste en menospreciar los individuos pequeños. Los estudios efectuados igualmente en ríos de montaña. encuentran una relación directa entre el aumento de eutrofización de aguas y los cuantitativos de quironómidos, por lo que en el aspecto de la reducida carga orgánica del río Olo, podemos concluir que estos organismos desempeñan aquí un papel secundario en la circulación de energía.

Crustácea: una de las más interesantes diferencias entre el río Olo y ecosistemas semejantes estudiados en el ya citado trabajo de Tánago y Jalón, consiste en la ausencia de Gammarus sp. y su sustitución por Pro-asellus gr. coxalis. Henry y Magniez (1983) consideran que existe una multitud de especies pertenecientes a este grupo en las zonas de influencia mediterránea de carácter oligohalino.

\section{Estructura de la comunidad}

Las variaciones biocenóticas que fueron mencionadas no son suficientes para considerar distintas comu- nidades de las tres estaciones ya que su correlación es altamente significativa para $\mathrm{P}=0.01$ (el coeficiente de Spearman, $r_{*}$, el valor de $t$ y el $n^{\circ}$ de especies usadas en la comparación se indican en la tabla 2). Tal hecho se debe a una nítida semejanza en lo que se refiere a la importancia relativa de los grupos dominantes. por lo que la biotipologia sólo es explicada por especies que presentan una composición relativa inferior. aunque no pueda ser despreciada. en este caso. la influencia causada por distintos micro-hábitats.

La macrofauna se presenta globalniente dividida por los varios grupos tróficos según los siguientes porcentajes (se siguió el mismo modelo de Tánago y Jalón (1982), en lo que se refiere a distribución de los taxones por diversos grupos): Predadores $\pm 5 \%$; Colectores i 50\%: Detritivoros $\pm 20 \%$ : Fitófagos i $15 \%$.

Esta composición trófica se nos figura natural dado que en el rhitron la proporción de materia orgánica que llega al río procedente de vegetación alóctona es generalmente mayor de la que tiene origen en el propio lugar (Mann. 1975), y es semejante a la obtenida para las mismas zonas ecológicas de la cuenca del Duero.

La diversidad en los varios puntos de muestreo y diferentes periodos (tabla 3) es de un modo general inarcadaniente superior a la que fue calculada por Tánago y Jalón. esclareciendo que el nivel de identificación fue semejante. Estos autores solamente \&tuvieron valores idénticos en las partes superiores de los ríos Arlanzón. Carrión, Durandón y Agueda durante el invierno. Las variaciones de $\mathrm{H}^{\prime}$ a lo largo del año y entre estaciones están ciertamente asociadas al tamaño de la muestra, como otros índices basados en la teoría de información (Wilhm, 1970) y con el nivel de identificación que debe ser idéntico para todos los grupos (Prat. et al.. 1983). lo que no se verificó en el caso presente

\section{Poblaciones de peces.}

He aqui los datos obtenidos durante el único muestreo

Estación 1.-Especies: Salmo tratta /ario-100\%" Biomasa hor $=30.4 \times 10^{3} \mathrm{~g}$

Estación 3.-Especies: (hondostroma polytepis polylepis 63.6\%: Salmo trutla farto 27.2\%: Lenciscus cephalus cabeda $9.2 \%$

Hiomasa ha- $: 4.2 \times 10^{3} \mathrm{~g}$.

Como se observa bajo el punto de vista piscícola. hay una considerable distinción en lo que se refiere al n." de especies y biomasa ha ${ }^{-1}$. Esta última es baja como sería de esperar en función de la reducida productividad de aguas poco mineralizadas. pero además pensamos que los valores más inferiores de la estación 3 sólo pueden ser explicados por la pesca excesiva que ahi ha tenido lugar. Valores semejantes a los 
obtenidos para la estación 1 son referidos por Valente (1984) en un río del norte de Portugal de idénticas características químicas y faunísticas, los cuales son, no obstante, nítidamente inferiores a los $123 \mathrm{Kg}$. hamencionados por Cuinat (1971) como biomasa media en los ríos franceses.

Como conclusión pensamos que algunas de las características peculiares exhibidas por el río Olo, cuando es comparado con otros cursos de agua de la cuenca del Duero, podrían ser debidas a la orientación
W-E que le confiere una exposición con marcada influencia marítima. Por otra parte la estructura biocenótica de las poblaciones de macroinvertebrados se mantiene relativamente semejante, o sea, en términos longitudinales como temporales, en lo que se refiere a la diversidad total y a la presencia de las especies dominantes, ciertamente como resultado de una estabilidad química perfectamente evidente la cual sobrepasa en influencia las posibles variaciones biológicas introducidas por el factoraltitud.

APENDICE

\begin{tabular}{|c|c|c|}
\hline ESTACION 1 & ESTACION 2 & ESTACION 3 \\
\hline $6 / 839 / 83 \quad 12 / 83 \quad 5 / 846 / 849 / 84$ & 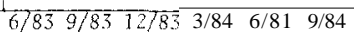 & $\begin{array}{llllll}67 \overline{8} & 9 / 8 & 12 / 83 & 1 / 84 & 6 / 84 & 9 / 84\end{array}$ \\
\hline
\end{tabular}

Tricladida

Polycelis fellina

Polycelis nigm

Polycelis nigrn

Polycelis tenuis

Dugesia gonocephala
Dugesia polychron

Gastropoda

Ancylus fluviatilis

cf. Ferrisiu sp.

Oligochaeta

Enchytracidae

Lumbricidae

Lumbricul idae

Naididae

Tubificidne

Hirudinea

Glossiphonia heteroclita

Glossiphonia ef .complanata

Helobdella stagnal is

Erpobdella cf. monostriata

Hydracarina

Isopodd

Pro-asellus gr. coxalis

Epheneroptera

Siphlorurus sp.

Raetis alpinus

Raetis cf. gr. alpinus

Baet is buceratus

Raetis fuscntus

Raetis rnelanony $x$

Raet is rhodani

leptageni idae

Feorus torrentim/sylvicola

Rhithrogena sp.

Ecdyonurus sp.

Heptagenia sulphurea

Ephemerella ignita

Ephemerella mesoleuca

Serratella a1bai

Euryophella iberica

Cnenis moesta

Calliarcys humi 1 is

Thraulus bellus

Paraleptophlebia cinct

thabroleptoides sp.

tabrophlebia fuscn

Dipterophlebiodess
Ephemera 1 ineü ta

Plecoptera

Brachyptera ef arcuata

Amphinemura standfussi

Anphinemura sulcicollis

Amphinemura triangularis

Semoura sp.

$\begin{array}{llll} & 5.9 & 1.1 & 0.7 \\ 2.1 & & & \\ 2.1 & & & \end{array}$

$\begin{array}{ll}2.2 & 0.7\end{array}$

0.5

$\begin{array}{lll}7.8 & 3.3 & 0.4\end{array}$

0.1
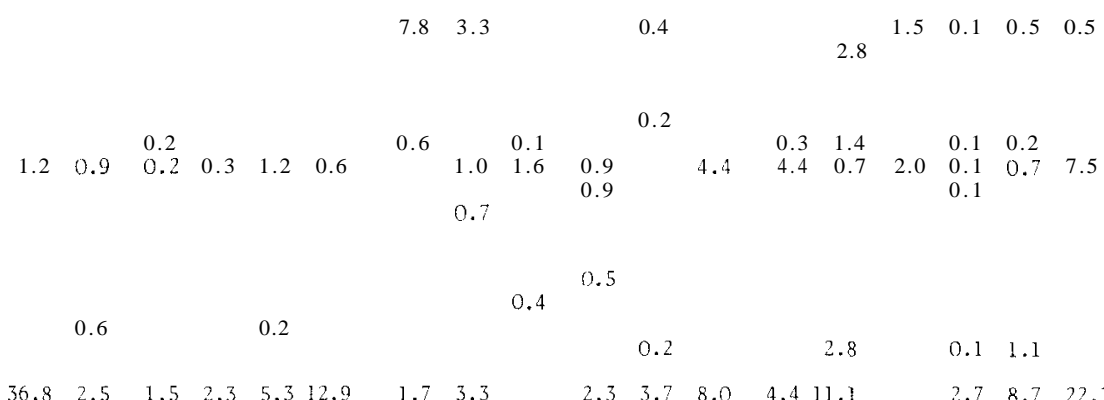

$\begin{array}{llllll}36.8 & 2.5 & 1.5 & 2.3 & 5.3 & 12.9\end{array}$

$\begin{array}{lllll}0.4 & 1.2 & 0.5 & 1.6 & 0.2\end{array}$

0.4

0.3

1.8

0.7

$0.6 \quad \begin{array}{ll}3.7 \\ 0.6\end{array}$

$\begin{array}{lllllll}3.3 & 1.6 & 2.2 & 8.7 & 4.6 & 1.7 & 8.4\end{array}$

$4.1 \quad 9.0 \quad 3.6$

$38.3 \quad 2.1$

0.1

0.8 


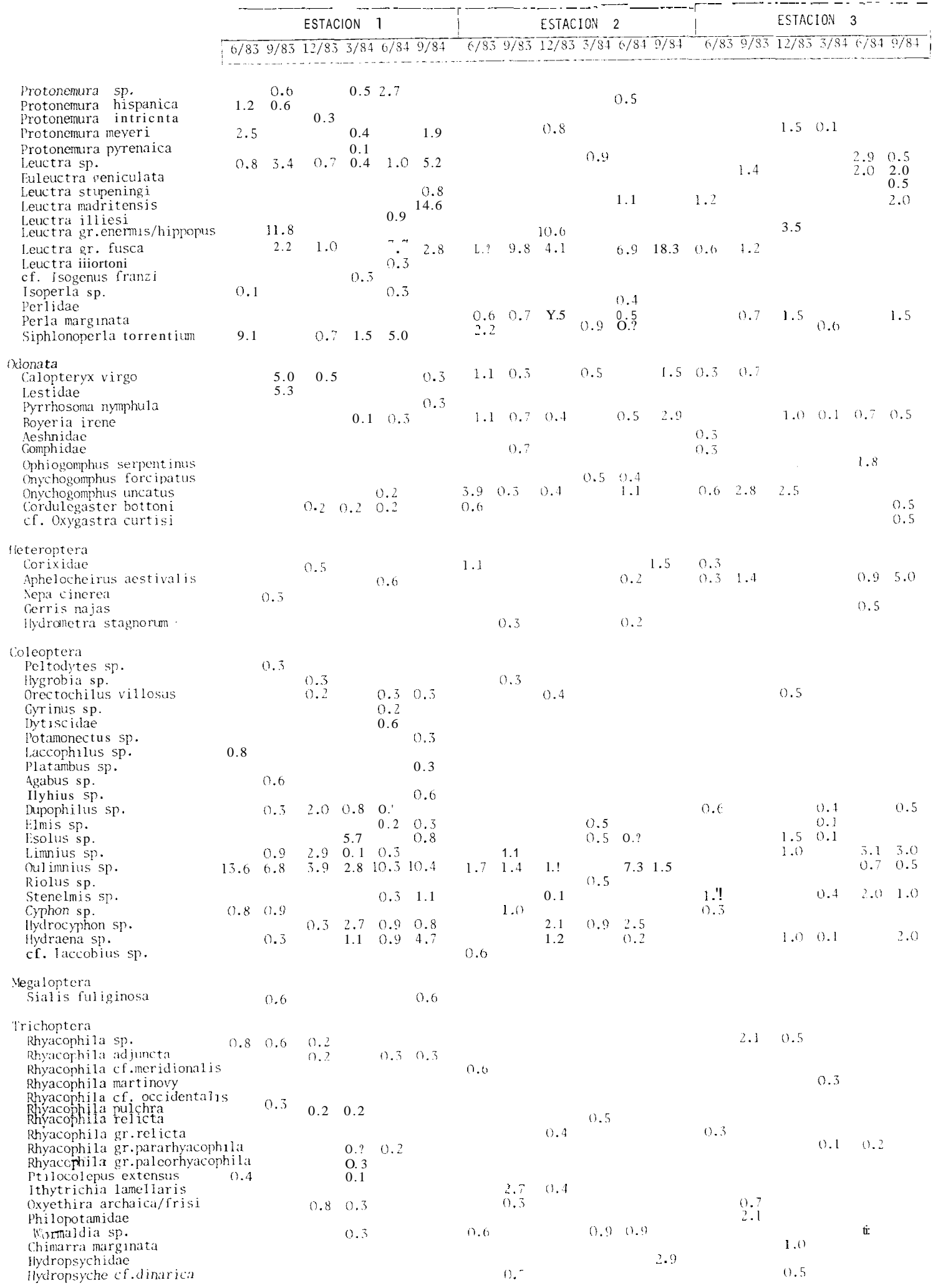




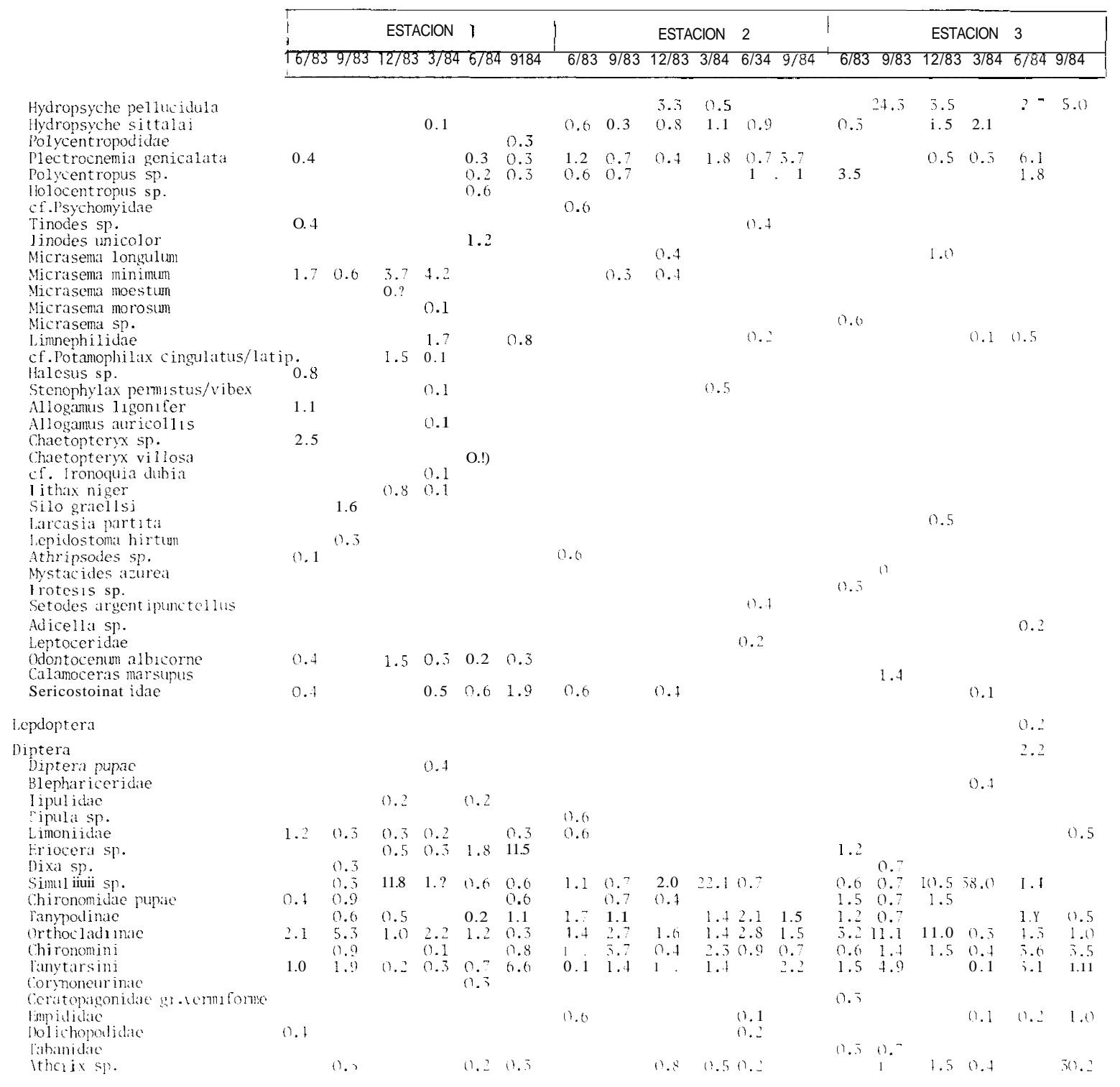

\section{BIBLIOGRAFIA}

Amanieu, M. \& Lassere, G. 1972. Ecologie des populations animales en milieu aquatique. L'niversití des Sciences et Techniques du Languedoc

Baguña, J.; Saló, F. \& Romero, R. 1982. Biogeografia de las pianarias de aguas dulces (Platelminthes. Turbellaria. Tricladida. Paludicola) en España. Datos preliminares. Acta. I Congr. Expr. Limnol. Prat N. ed.. 165-180.

Hoon, P.J. 1979. Studics on the spatial and temporal distribution of larval Hydropsychidae in the North Tyne river system (Northern England). $1 r(h . M y d r o-$ biol. 85 (3):336-359.

Cortés, R. 1981. Estudo eco-hidrobiologico do rio Sordo. Instituto Universitario de Tras-os-Montes e Alto Douro, Vila Real. 100 pp.

Cuinat, R. 1971. Principaux caractéres démographiques observés sur 50 riviéres a truites francaises. Influence de la pente et du calciuni. Ann/s. Limnol. 2 (2): 187-207.

Décamps, H. 1970. Les larves de Brachycentridae (Trichoptera) de la faune de France. Taxonomie et ecologie. Annls. Limnol. 6, 51-73.

Fontoura, A.P. \& Moura, A.M. 1984. Effects of some industrial effluents on the biological quality of the water of the river Lima. Publ. Inst. Zool. Faculd. Cienc. Porio. 
Gudicelli, J.; Dia, A.; Legier, P. 1980. Etude hydrobiologique d'une riviére de région méditerranéenne, L'Argens (Var, France). Bijdr. tot Dierk. 50 (2): 301-341.

Henry, J.P.; Magniez, G. 1983. Crustaces Isopodes Bull. Soc. Limn. Lyon. 52 (10):319-357.

Illies, J. \& Botosaneanu, L. 1963. Problémes et méthodes de la clasification et de la zonation écologique des eaux courantes considerées surtout du point de vue faunistique. Mitt. Int. Verein. Lirnnol., 12: 1-57.

Jalón, D.G. 1981. La larva de Rhyacophila adjuncta Mc Lachlan 1884 (Trich. Rhyacophilidae). Bol. Assoc. Esp. Entorn., 5: 25-29.

Jalón, D.G. \& Tánago, M.G. 1982. Introducción a una Zoosociología del macrobenthos en los ríos de la Sierra de Guadarrama. Bol. Est. Cent. Ecol.. 11 (21): 63-7 1.

Lapchin, L. \& Neveu, A. 1979. Ecologie des principaux invertebrés filtreurs de la Basse Nivelle (Pyrenées Atlantiques). Annls. Limnol., 15(2): 139-1 53.

Legier, P. 1972. Repartition et etagement de l'habitat chez les planaires triclades d'eau douce en Provence Occidentale. Annls. Limnol., 8: 31-40.

Mann, K.H. 1975. Patterns of energy flow. In: River Ecology Whitton, B.A. (eds.), 248-263. Blackwell, Scientific Publ. 725 pp.

Pattee, E. \& Gourbault, N. 1981. Turbellaires Tricladides Paludicoles (planaires d'eau douce). Bull. Soc. Lymn. Lyon. 50 (9):279-304.

Peña, G.G. 1982. Factores que determinan la distribución de los simulídios y quironomídcos (Diptera) de la red hidrográfica andorrana. Actas I Congr. Esp. Lirnnol. Prat N. (ed.),197-205.

Prat et al., 1983. Mem. Am. Ent. Soc. 34.

Puig, M.A. 1984. Distribution and ecology of the stoneflies (Plecoptera) in Catalonian rivers ( $N E-S p a i n)$. Annls. Limnol. 20(1-2): 75-80.
Quero, A.V. 1982. Estudio de las larvas de Chironomidae (Diptera)en el río Cubillas (Granada). Actas I Congr. Esp. Limnol. Prat, N. (ed.):207-211.

Resh, V.H. 1979. Sampling variability and life history features:basic considerations in the design of aquátic insect studies. J. Fish Res. Board. Can., 36: 290-311.

Shannon, C.E. \& Weaver, W. 1949. The mathematical theory of communication. The University of Illinois Press: Urbana: 19-27.

Sousa, E.; Fernandes, J.; Ferreira, 'T.; Andrade, I.; Brito, F.; Janeiro, A. 1984. Esaio de caracterizaçao da qualidade da água num efluente do Guadiana. Simp. Prot. Zonas Ribeirinhas.

Tánago, M.G. \& Jalón, D.G. 1982. Estudio para una metodología de clasificación biotipológica de los ríos españoles: aplicación a la cuenca del Duero. Trab. Ceotma, Mopu, Madrid. $368 \mathrm{pp}$.

Tánago, M.G. 1984a. Contribution of the zoogeography of the Spanish Ephemeroptera. Proc. Ivth. Intern. Confer. Ephemeroptera Landa, V. et al. (cds.): $135-145$.

'Tánago, M.G. 1984b. Distribución y biología de la familia Baetidae (Ephem.) en la Cuenca del Duero. Bol. Assoc. Esp. Entom. 8: 73-94.

Valente, A. 1984. Primeiros dados do estudo da populaçao de Salmo trutta L. num. pequeno rio do Norte de Portugal. Publ. Inst. Zool. Faculd. Cienc. Porto. $15 \mathrm{p}$.

Vernaux, J. \& Faessel, B. 1976. Larves du genere Hydropsyche (Trichopteres Hydropsychidae). Taxonomie donnés biologiques et écologiques. Ann/s. Limnol., 12(1): 7-16.

Wilhm, J.L. 1970. Rangc of diversity index in benthic macroinvertebrate populations. Journal WPCF. 42(5): $221-224$. 